\section{Recent progress of the Golgi technique and electron microscopy to examine dendritic pathology in Alzheimer's disease}

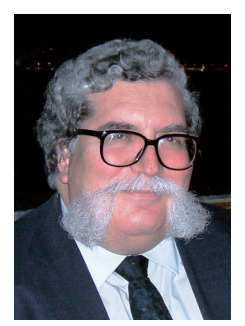

\author{
"Golgi study also provides an insight into the \\ pathogenesis of neurological disorders and the \\ mechanisms involved in the remodeling of \\ neuronal networks in the recovering brain."
}

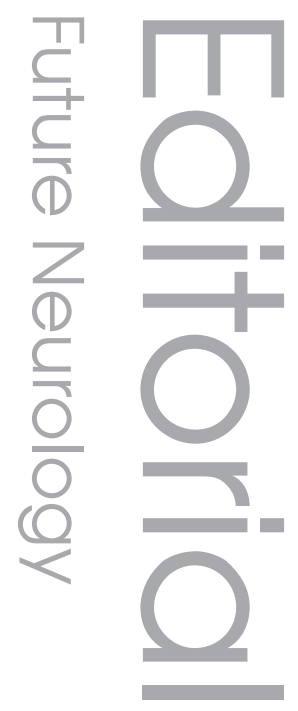

Stavros J Baloyannis

Aristotelian University, Research Institute for Alzheimer's Disease, Angelaki 5, 54621 Thessaloniki, Greece - Tel.. +30 2310270434 - Fax: +30 2310270434 m sibh844@otenet.gr

Some old methods, frequently used in neuropathology in the past, remain to be important in attempting to proceed to detailed morphometric estimations and morphological descriptions of neurons, axons, dendrites and neuronal circuits in health and disease.

The silver impregnation technique is a very efficient method for visualizing whole neurons and neuronal circuits in a pictorial appearance. The technique, which is more than 100 years old, was introduced by Golgi as 'reazione nera' (black reaction) [1] and was applied extensively by Ramon y Cajal in describing many types of neurons in the brain; he documented the fascinating appearance of the neuronal circuits in the histological organization of the nervous system in his 'neuronal doctrine' [2].

For many years, the Golgi method has been considered to be the classical technique for the precise description of neurons, since it delineates the profile of neurons and dendritic ramifications with unique clarity, which displays the dendritic arborization completely. Gradually, the term 'Golgi technique' has started to refer to a group of heavy metal impregnation methods that rely on preparation of neural tissue with potassium dichromate [3].

Several modifications of this technique have been introduced by various laboratories, such as the modified Golgi stain, rapid Golgi technique, modified Golgi-Cox method, Golgi-Braitenberg method and many others, which have contributed to optimizing the quality of impregnation, making staining faster, easier, brighter, richer, less patchy and evenly dispersed [3,4].

Currently, despite the delicate nature and the unpredictability of Golgi techniques, they remain virtually unique for demonstrating neurons and dendritic morphology in a biplanar or stereoscopic way, viewed either by conventional or confocal light microscopy, equipped with a computerized image-analysis system. Golgi techniques, associated with electron microscopy, have broadened our views on neuronal development, maturation, plasticity, senescence and degenerating processes.

Dendritic pathology and the decrease of dendritic spine density are prominent findings in many neurological and psychiatric disorders. The pathology of dendritic spines mostly consists of altered spine numbers, distorted spine shapes, giant spines, alteration of organelles within the spines and abnormal spine protrusion. Relevant morphological alterations have been described in epilepsy, Huntington's disease, Parkinson's disease, prion diseases and schizophrenia [5]. Nevertheless, dendritic and spine pathology have also been described in autism, drug addiction, brain trauma and in some genetic disorders such as Down's syndrome and fragile X syndrome [6].

Thus, Golgi techniques have traditionally been applied in order to visualize dendritic spines, which represent the smallest, most sensitive and vulnerable structural unit of the neuron, which are involved in many diseases of the brain $[5,6]$. Golgi study also provides an insight into the pathogenesis of neurological disorders and the mechanisms involved in the remodeling of neuronal networks in the recovering brain.

In Alzheimer's disease, which is the most frequent age-dependent dementia, dendritic and spinal pathology play very important roles in the decline of mental faculties, since spines and synapses are very dynamic structures, expressing plasticity, which is indispensable in learning and memory.

\section{Keywords}

- Alzheimer's disease

- dendrite = dendritic spine

- electron microscopy = Golgi

staining $=$ synapse

Future Medicine part of 
Although neurofibrillary tangles and neuritic plaques have unanimously been considered to be the hallmarks of Alzheimer's disease, dendritic and synaptic alterations are also prominent at the early stages of the disease $[7,8]$. Studies based on Golgi staining and electron microscopy have revealed dendritic and spinal changes in the prefrontal cortex, cerebellum and visual and acoustic cortices [9,10], which consisted mostly of a tremendous decrease of spine density, spinal poverty and loss of the distal dendritic spines. In addition, the morphometric estimation of the dendritic branches and spines of early cases of Alzheimer's disease revealed a $45 \%$ reduction of branches of the apical and the basal dendrites and a $52 \%$ reduction of dendritic spines in the acoustic and visual cortices [11].

\section{"...Golgi techniques ... remain virtually unique for demonstrating neurons and dendritic morphology in a biplanar or stereoscopic way..."}

A study of the vestibulo-cerebellar system, based on the Golgi technique and electron microscopy, revealed a tremendous loss of dendritic branches and spines of Purkinje cells in the nodule and flocculus [12]. Electron microscopy revealed substantial synaptic alterations between mossy fibers and granule cell dendrites, characterized by polymorphisms, paucity of synaptic vesicles and marked mitochondrial alterations [12]. In the cerebellar cortex, morphometric estimation revealed a $43 \%$ reduction of Purkinje cell dendritic spines and a $16 \%$ increase in the length of the spines, associated with marked abridgment of dendritic arborization, which covered only $61 \%$ of the depth of the molecular layer [11].

It is important to emphasize that a substantial body of evidence based on Golgi techniques and electron microscopy confirmed that dendritic and spinal pathology is not related to neuritic plaques, since the cerebellum is virtually free from typical Alzheimer's pathology despite the numerous dendritic and spinal changes of Purkinje cells [12]. In addition, synaptic loss and microglia proliferation seem to precede tau pathology in animal models [13]. The application of the Golgi-Nissl method for the study of medial geniculate bodies and the inferior colliculi in Alzheimer's disease revealed a marked neuronal loss, substantial abbreviation of the dendritic arborization and a decrease in spine density, whereas Bodian staining did not reveal any Alzheimer's pathology [14].
Golgi techniques have revealed that CajalRetzius cells, which are among the earliest neurons to be generated in the cerebral cortex, disappear or show a dramatic shortening of dendrites in early cases of Alzheimer's disease [15], an observation that pleads reasonably in favor of their possible implication in the pathogenetic pathways of Alzheimer's disease. The loss of Cajal-Retzius cells may play a substantial role in the disruption of the microcolumnar ensembles of the cortex, also affecting the synapses, owing to their crucial contribution in harmonious synaptogenesis.

In an experimental study on mice with accelerated senescence, the use of Golgi staining revealed that the dendritic spines started to decrease as the age of the animals advanced and their memory declined [16].

Dendritic changes and axonopathy are presumably linked to diabetic encephalopathy, age-related neurodegenerative disorders and normal aging; they may also be involved in the labyrinth of the pathogenesis of Alzheimer's disease. In a recent study on a diabetic rat model, swelling of axons and varicosities of dendrites were described in the cortex, associated with hyperphosphorylated tau, also seen at the early stages of Alzheimer's disease [17].

\section{"...dendritic and spinal pathology is not related to neuritic plaques..."}

The visualization of dendrites and dendritic spines by the high-quality Golgi-Cox technique, in combination with immunocytochemical procedures, viewing in confocal microscopy, is a method of fundamental importance in neuroscience, since it allows simultaneous appearance of the dendritic arborization and its antigens' characterization, in a $3 \mathrm{D}$ way, facilitating multiple analyses in experimental models [18].

The cause of Alzheimer's disease remains enigmatic. However, the study of the neuronal circuits, the distribution of synapses and the detailed analysis of the presynaptic and postsynaptic components, implying electron microscopy, is very important for tracing the early disruption of the neuronal circuits and the advancing synaptic pathology. In addition, a substantial body of evidence underlines the possible implication of the mitochondria and Golgi apparatus in the pathogenesis of neurodegenerative disorders, including Alzheimer's disease, that are well documented by electron microscopy, which is the gold 
standard for the morphological estimation and quantification of the organelles in the whole neuron [19-21].

Recent studies have also revealed the molecular mechanisms underlying synapse and spine pathology $[8,22,23]$. Studies on cell cultures, exposed to oligomers of $\beta$-amyloid peptide, which combined immune staining and electron microscopy, revealed that a fraction of neurons demonstrate increased tau phosphorylation, redistribution of tau into neuronal soma and dendrites, local elevation of calcium, loss of spines, pronounced depletion of cytoskeleton and a depletion of mitochondria $[8,22,23]$. It should be emphasized that mitochondrial pathology is particularly obvious in dystrophic dendritic profiles and correlates substantially with the morphological and morphometric alteration of the spines [19].

Serial-section electron microscopy is the ideal method for the quantification of the synapses, but it is a time-consuming technique. The recent development of automatic electron microscope methods, based on the combination of focused ion beam milling and scanning electron microscopy, is an excellent tool for describing the morphology and the spatial distribution of synapses in different areas of the brain in the study of Alzheimer's disease. This technique can evaluate the ultrastructural alterations of the synapsis and quantify them per volume [24]. In addition, by virtue of stereological methods, the study and the evaluation of the synaptic organization in space is also feasible.

Even today, silver impregnation techniques and electron microscopy offer many capabilities for morphological and morphometric study of the brain in aging [25] and Alzheimer's disease. Apart from the staging of Alzheimer alterations by Golgi techniques [26], silver impregnation in association with electron microscopy enables the detailed analysis of dendritic pathology, visualizing and quantifying the dramatic changes of dendritic spines in size, shape and interior organization. This provides a very thorough idea of the sequence of alterations occuring in neurons, dendrites and dendritic spines as Alzheimer's disease advances, and also delineates the roles that mitochondria and Golgi apparatus may play in dendritic and synaptic pathology. Strategies protecting the integrity of mitochondria may be beneficial in Alzheimer's disease. Therapies targeting basic processes, such as energy metabolism, free-radical generation or specific interactions of disease-related proteins with mitochondria may be very promising. In parallel, on the basis of dendritic pathology, new strategies protecting dendrites and enhancing synaptogenesis may also have a substantial effect in the treatment of early cases of Alzheimer's disease.

\section{Financial \& competing interests disclosure}

The author has no relevant affiliations or financial involvement with any organization or entity with a financial interest in or financial conflict with the subject matter or materials discussed in the manuscript. This includes employment, consultancies, honoraria, stock ownership or options, expert testimony, grants or patents received or pending, or royalties.

No writing assistance was utilized in the production of this manuscript.

\section{References}

1. Golgi C. On the structure of nerve cells 1896. J. Microsc. 155, 3-7 (1969).

2. Ramon y Cajal S. Neuron Theory or Reticular Theory? Objective Evidence of the Anatomical Unity of the Nerve Cells. Consejo Superior de Investigaciones Cientificas, Madrid, Spain (1954).

3. Scheibel ME, Scheibel AB. The methods of Golgi. In: Neuroanatomical Research Techniques. Robertson RT (Ed.). Academic Press, NY, USA, 90-114 (1978).

4. Levine ND, Rademacher DJ, Collier TJ et al. Advances in thin tissue Golgi-Cox impregnation: fast, reliable methods for multi-assay analyses in rodent and nonhuman primate brain. J. Neurosci. Methods 213(2), 214-227 (2013).

5. Fiala JC, Spacek J, Harris KM. Dendritic spine pathology: cause or consequence of neurological disorders? Brain Res. Rev. 39, 29-54 (2002).

6. Penzes P, Cahill ME, Jones KA et al. Dendritic spine pathology in neuropsychiatric disorders. Nat. Neurosci. 14, 285-293 (2011).

7. Terry RD, Katzman R. Senile dementia of the Alzheimer type. Ann. Neurol. 14, 497-506 (1983).

8. Wendou Y, Bingwei L. Synapses and dendritic spines as pathogenic targets in Alzheimer's disease. Neural Plast. 2012, 247150 (2012).

9. Baloyannis SJ. Dendritic pathology in Alzheimer's disease. J. Neurol. Sci. 283, 153-157 (2009).

10. Baloyannis SJ, Costa V, Mauroudis I et al. Dendritic and spinal pathology in the acoustic cortex in Alzheimer's disease: morphological and morphometric estimation by Golgi technique and electron microscopy. Acta Otolaryngol. 127, 351-354 (2007).
11. Mavroudis IA, Fotiou DF, Adipepe LF et al. Morphological changes of the human purkinje cells and deposition of neuritic plaques and neurofibrillary tangles on the cerebellar cortex of Alzheimer's disease. Am. J. Alzh. Dis. Oth. Dement. 25, 585-591 (2010).

12. Baloyannis SJ, Manolidis SL, Manolidis LS. Synaptic alterations in the vestibulocerebellar system in Alzheimer's disease - a Golgi and electron microscope study. Acta Otolaryngol. $120,247-250$ (2000).

13. Yoshiyama Y, Higuchi M, Zhang B et al. Synapse loss and microglial activation precede tangles in a P301S tauopathy mouse model. Neuron 53, 337-351 (2007).

14. Baloyannis SJ, Mavroudis I, Manolidis SL, Manolidis LS. Synaptic alterations in the medial geniculate bodies and the inferior colliculi in Alzheimer's disease: a Golgi and 
electron microscope study. Acta Otolaryngol. 129(4), 416-418 (2009).

15. Baloyannis SJ. Morphological and morphometric alterations of Cajal-Retzius cells in early cases of Alzheimer's disease: a Golgi and electron microscope study. Intern. J. Neurosci. 115, 965-980 (2005).

16. del Valle J, Bayod S, Camins A et al. Dendritic spine abnormalities in hippocampal CA1 pyramidal neurons underlying memory deficits in the SAMP8 mouse model of Alzheimer's disease. J. Alzheimers Dis. 32(1), 233-240 (2012).

17. Zhou Y, Luo Y, Dai J. Axonal and dendritic changes are associated with diabetic encephalopathy in rats: an important risk factor for Alzheimer's disease. J. Alzheimers Dis. 34(4), 937-947 (2013).

18. Spiga S, Acquas E, Puddu MC, Mulas G, Lintas A, Diana M. Simultaneous Golgi-Cox and immunofluorescence using confocal microscopy. Brain Struct. Funct. 216(3), 171-182 (2011).

19. Baloyannis SJ. Mitochondrial alterations in Alzheimer's disease. J. Alzh. Dis. 9(2), 119-126 (2006).

20. Baloyannis $S$. The Golgi apparatus of Purkinje cells in Alzheimer's disease. In: Neuropathology Back to the Roots. Bohl J (Ed.). Shaker Vertag, Aachen, Germany, 1-10 (2002).

21. Calkins MJ, Manczak M, Mao P, Shirendeb $\mathrm{U}$, Reddy PH. Impaired mitochondrial biogenesis, defective axonal transport of mitochondria, abnormal mitochondrial dynamics and synaptic degeneration in a mouse model of Alzheimer's disease. Hum. Mol. Genet. 20(23), 4515-4529 (2011).

22. Zempel H, Thies E, Mandelkow E, Mandelkow EM. Abeta oligomers cause localized $\mathrm{Ca}(2+)$ elevation, missorting of endogenous tau into dendrites, tau phosphorylation, and destruction of microtubules and spines. J. Neurosci. 30(36), 11938-11950 (2010).

23. Adalbert R, Coleman MP. Axon pathology in age-related neurodegenerative disorders. Neuropathol. Appl. Neurobiol. doi:10.1111/j.1365-2990.2012.01308.x. (2012) (Epub ahead of print).

24. Blazquez-Llorca L, Merchán-Pérez A, Rodríguez R, Gascón J, Defelipe J. FIB/SEM technology and Alzheimer's disease: three-dimensional analysis of human cortical synapses. J. Alzheimers Dis. 34(4), 995-1013 (2013).

25. Mavroudis I, Petrides F, Manani M et al. Dendritic and spinal alterations of the spiny stellate cells of the human visual cortex during normal aging. Folia Neuropathol. 50(3), 261-269 (2012).

26. Braak H, Braak E. Neuropathological stageing of Alzheimer-related changes. Acta Neuropathol. 82, 239-959 (1991). 